\title{
REA para el aprendizaje a distancia que promueva la motivación y la autonomía.
}

\author{
OEP to promote motivation and autonomy in distance learning \\ Ana M. Mendoza-Batista ${ }^{a}$,Vilma A. Vázquez-Morales ${ }^{b}$
}

\begin{abstract}
:
This research explores how implementing some OER promoted motivation and autonomy in online courses. This descriptive research highlighted the execution of various OER among 50 undergraduate students. The survey data showed students' perceptions and focused on three main traits: likeness, utility, and preference. The results showed a positive response and the pertinence in usage in online courses.
\end{abstract}

\section{Keywords:}

OER, distance learning, Flipgrid, Quizizz, Edpuzzle, Padlet.

\section{Resumen:}

Esta investigación explora cómo la implementación de algunos recursos educativos abiertos promueven la motivación y la autonomía en cursos a distancia. Esta investigación descriptiva destaca la aplicación de varios de estos recursos en 50 estudiantes de licenciatura. Los datos en la encuesta muestran las percepciones de los estudiantes y se enfoca en tres características principales: gustos, utilidad y preferencia. Los resultados muestran una respuesta favorable y la pertinencia de su uso.

\section{Palabras Clave:}

REA, aprendizaje a distancia, Flipgrid, Edpuzzle, Padlet

\section{INTRODUCCIÓN}

A lo largo de nuestra época, la enseñanza y aprendizaje del idioma inglés ha estado sujeto a un proceso de cambio y adaptación como resultado de la constante evolución de nuestra sociedad en ámbitos culturales, sociales, económicos y tecnológicos. En este tenor, la enseñanza se ha tenido que transformar a partir de nuevas necesidades por y para la sociedady da cabida a nuevas corrientes de aprendizaje y transformación de habilidades de aprendizaje del siglo XXI.

Es decir, que habilidades debe adquirir los estudiantes para el savoir-faire y tener éxito en sus disciplinas en esta nueva era de la información.

Entendemos la enseñanza como un conjunto de actividades y acciones tendientes a formar ciudadanos, luego entonces debe funcionar como un mecanismo de cambio y transformación. En un mundo globalizado, la enseñanza de lenguas sirve como un puente de unión entre dos culturas y da amplitud a la multiculturalidad y la cohesión social (Byram y Fleming, 2001, 10).

La enseñanza de idiomas ha hecho sus propios avances de acuerdo a los cambios que han existido en la sociedad. Son muchos los soportes utilizados por los profesores de una lengua extranjera: pasando por el libro de texto, los laboratorios de idiomas, la televisión, audiocassettes, hasta la llegada de las TICs. En este sentido, el uso de la tecnología en la educación ha propiciado que los estudiantes y profesores tengan un número ilimitado de opciones para el aprendizaje, ya que los estudiantes están interactuando con la tecnología constantemente fuera del aula, se están creando nuevos entornos y ambientes educativos o de aprendizaje (Vite,H.R 2012).

Redimensionar los ambientes educativos en la escuela implica, además de modificar el medio físico, los recursos y materiales con los que se trabaja, un replanteamiento de las acciones educativas que en ella se desarrollan y que posibiliten el encuentro de los actores, dando pie a materiales y actividades que estimulen la curiosidad, la capacidad creadora, el diálogo,

\footnotetext{
${ }^{a}$ Universidad Autónoma Metropolitana, Centro de Enseñanza de Lenguas Extranjeras, https://orcid.org/0000-0002-8807-1481 Email: amendoza.batista@izt.uam.mx

b Universidad Veracruzana, Centro de Idiomas - Región Veracruz, https://orcid.org/0000-0002-4696-3836, Email: vilvazquez@uv.mx
} 
intereses, necesidades y estados de ánimo. Para los nuevos profesores y estudiantes, la tecnología se considera una necesidad básica que se debe implementar en las aulas.

Y aunque la tecnología en las aulas es meramente para dar apoyo al nuevo paradigma de enseñanza, Prensky (2008) asevera que la tecnología coadyuva de manera eficiente a motivar y potencializar el aprendizaje en nativos digitales. Pizarro \& Cordero (2013), postulan que las TICs ayudan al proceso de enseñanza aprendizaje transformando a los estudiantes en aprendientes activos y auto constructores de su propio conocimiento. Dommus (2010) afirma que cada vez más los docentes están observando las bondades del uso de la tecnología como recurso en la clase de lengua ya que habilita el aprendizaje independiente y colaborativo en los cuales el estudiante puede adquirir y practicar los nuevos conocimientos en la lengua meta. A raíz de la pandemia Covid 19 en este año, se hace un cambio forzado de la clase presencial al formato online, y el uso de de la tecnología cobra más relevancia, es entonces primordial la aplicación de nuevas estrategias de Enseñanza-Aprendizaje. El profesor por su parte se ve en la necesidad de adquirir, diseñar y adaptar materiales que permitieran a los estudiantes utilizarlos de forma independiente.

\section{OBJETIVO}

El objetivo de este trabajo es dar a conocer los recursos que han sido más significativos y que coadyuvaron a la motivación y el aprendizaje autónomo en los estudiantes durante esta contingencia.

\section{MARCO TEÓRICO}

En los últimos años, los profesores han empleado recursos tecnológicos como sustituto del material impreso tradicional (Cassany D., 2008) El uso de la tecnología en la clase de lenguas extranjeras es una necesidad ya que tiene muchos beneficios como: hacer el proceso de enseñanza-aprendizaje más disfrutable; motivando tanto para los alumnos como a los docentes. Los recursos multimedia utilizados pueden ser variados y atractivos además de encontrarse una gran gama de materiales auténticos lo cual propicia el aprendizaje centrado en el alumno y la autonomía (Davies \& Hewer ,2012; Nomass, 2013).

La tecnología coadyuva a la autodirección permitiendo que los estudiantes planifiquen, supervisen y reflexionen sobre su propio aprendizaje, evidenciando su progreso, compartan conocimientos y presenten soluciones creativas. En este sentido, el uso de recursos educativos abiertos permean y posibilitan todas estas acciones.

\section{RECURSOS EDUCATIVOS ABIERTOS}

Los Recursos Educativos Abiertos (REA) o Open Educational Resources (OER) por sus siglas en inglés son herramientas esenciales para apoyar al docente frente al nuevo proceso de enseñanza aprendizaje de los nativos digitales. (Prensky, 2018). Los docentes pueden tener un gran apoyo para usar en sus aulas de manera sincrónica o asincrónica, según sea sus necesidades. Estos REA son definidos por Miao, F. Mishra, S \& McGreal, R. (2016) de la UNESCO como, "materiales didácticos, de aprendizaje o investigación que se encuentra en el dominio público o que han sido publicados con una licencia de propiedad intelectual que permite su utilización adaptación y distribución gratuita" (pág. 8). Estos recursos educativos abiertos permiten cinco libertades reconocidas como las cinco R: retener, reutilizar, revisar, recombinar, $\mathrm{y}$ redistribuir: permitiendo a los docentes ahorrar tiempo y esfuerzo en la elaboración de recursos (Wiley D, 2014).

Los REA al ser atractivos y significativos para los estudiantes promueven la motivación, la cual se define según la Real Academia de la Lengua Española como un conjunto de factores internos o externos que determinan en parte las acciones de una persona. Gardner (1985) afirma que la motivación de los estudiantes está compuesta de tres elementos: esfuerzo, deseo y sentimiento; incrementando la motivación intrínseca y haciendo uso de la motivación instrumental.

\section{MOTIVACIÓN INTRÍNSECA E INSTRUMENTAL}

La motivación es un elemento fundamental para conseguir la autonomía, la cual se define como un proceso donde el estudiante autorregula su aprendizaje y toma conciencia de sus propios procesos cognitivos y socioafectivos a lo que se le llama metacognición. El estudiante autónomo debe tener la habilidad de tomar las riendas de su propio aprendizaje tomando las decisiones concernientes a todos los aspecto de su aprendizaje; como determinar objetivos, seleccionar estrategias y recursos; reflexionando y autoevaluando su propio aprendizaje (Garcia, 2012). Los estudiantes deben estar altamente motivados y ser proactivos para conseguir la autonomía.

Por otro lado, la motivación intrínseca está asociada a los sentimientos propios del estudiante y considera cómo los aprendientes se involucran en las tareas. Castillo et al (2019) sugieren que el aprendiente que está intrínsecamente motivado aprende bien y tienen mayores logros. Larrenua (2014) afirma que la motivación intrínseca está basada en la autonomía y competencia provocando la satisfacción y el disfrute con los objetivos logrados. Por otro lado, la motivación instrumental es la base de la meta para obtener alguna recompensa social o económica a través del logro de la segunda lengua, (Gardner and Lambert, 1959) refiriéndose así a algo más funcional para el aprendizaje de la lengua. Por lo tanto, mientras que los estudiantes intrínsecamente motivados quieren alcanzar una meta para su propia satisfacción, la motivación instrumental se 
utiliza como una herramienta para conseguir un objetivo externo.

Kazarian (2014) afirma que es necesario incentivar y mantener la motivación por aprender inglés partiendo de la premisa de que una enseñanza amena y atractiva es eficaz para promover el aprendizaje, involucrando a los estudiantes por actividades que impliquen un reto, disminuyan la ansiedad, desarrollen la autoconfianza e incrementen la curiosidad. Como resultado, los recursos utilizados en esta investigación fueron Kahoot, Quizizz, Flipgrid, Padlet, Quizlet y Edpuzzle. Estos REA son de uso común pero no conocidos por muchos profesores y fueron elegidos debido a que respondieron a las necesidades de los profesores y alumnos.

\section{EDPUZZLE}

Los videos son herramientas muy atractivas donde se combina el audio, elementos visuales y textos, permitiendo compartir contenidos de manera rápida y eficiente brindando a los estudiantes la posibilidad de ver y escuchar el material las veces que sea necesario. Edpuzzle es un REA que favorece personalizar contenido multimedia de acuerdo con las necesidades y los objetivos planteados por los docentes usando los videos de los canales más populares y adaptarlo para crear una actividad interactiva, que desarrolla la comprensión auditiva. Edpuzzle permite ajustar la longitud del vídeo e insertar preguntas de opción múltiple, preguntas abiertas y notas tanto orales como escritas para agudizar la comprensión auditiva y reforzar los conocimientos de algún tema ya que da retroalimentación instantánea, permitiendo al aprendiente auto evaluar su desempeño. https://edpuzzle.com/

\section{PADLET}

Padlet es una herramienta que contribuye a construir un espacio colaborativo de forma dinámica. Es un mural o póster interactivo que permite publicar, almacenar y compartir recursos multimedia de diferentes fuentes, de manera individual o en colaboración con un grupo de personas. Puede utilizarse tanto en el aula, o en la educación en línea, y se puede compartir en cualquier entorno virtual. Se pueden realizar actividades como: e-portafolios, colecciones creativas, anuncios, ejercicios de escritura libre, rincones didácticos, bibliotecas virtuales, galerías, videotecas, entre otros. https://padlet.com/

\section{FLIPGRID}

Flipgrid es una plataforma incluyente de aprendizaje social que permite a profesores y estudiantes interactuar utilizando vídeo (Baragar, 2020. p.1). Otros beneficios de este REA son que está centrado en el estudiante; desarrolla su capacidad de expresión, permite elaborar cuestionarios, grabar opiniones y respuestas de los alumnos, involucra estudiantes tímidos e introvertidos y les da confianza, provee al profesor de retroalimentación casi inmediata sobre lo aprendido, aumenta la posibilidad de análisis sobre un tema, democratiza el aprendizaje ya que empodera al estudiante al darle voz y voto (Murray, 2018). https://info.flipgrid.com/

\section{GAMIFICACIÓN}

El juego ha sido un recurso muy utilizado por los docentes para incrementar la motivación, la competencia y la emoción en el aprendizaje. La gamificación, como en la actualidad se le conoce, se refiere al uso de elementos de diseño del juego en contextos de no juego (Deterding, et al 2011:2) y pretende incorporar elementos lúdicos en áreas que no sean de juego. Busca incrementar el interés en los participantes, intenta satisfacer algunos de los deseos o necesidades humanas fundamentales, tanto del mundo real como del virtual, tales como: el reconocimiento, la recompensa, el logro, la competencia, la colaboración, y la autoexpresión (González et al, 2015).

\section{KAHOOT Y QUIZIZZ}

Para el aprendizaje de una lengua extranjera existen varios REA que apoyan la teorías de gamificación para apoyar el proceso de aprendizaje incrementando el interés y lograr que los estudiantes realicen ciertas acciones de manera satisfactoria.Algunos de estos recursos gamificables utilizados en el aula son: Kahoot, Quizizz y Quizlet.

Kahoot y Quizizz son juegos de preguntas con respuesta de opción múltiple o completar la frase. Ambos se pueden jugar a distancia individualmente o en equipos, ser asignados como tareas, y contienen colores, imágenes, música atractiva, retroalimentación instantánea y sistema de puntuación. Al ser una competencia entre los integrantes del grupo permite al estudiante tener mejor desempeño.

https://kahoot.it/ https://quizizz.com/

\section{QUIZLET}

Por otra parte, Quizlet presenta tres juegos, relacionar imagen con palabra o definición con palabras. Gravedad que es un juego donde el estudiante tiene que escribir la palabra antes de que el asteroide se estrelle en la tierra y por último "En Vivo"; donde los aprendientes trabajan colaborativamente para terminar sus palabras antes del otro equipo. Estos tres recursos han tenido gran aceptación en los estudiantes ya que implica el reto, la participación activa, socialización, la competencia, adquisición de conocimientos y habilidades, cambiando actitudes como lo menciona Kapp (2012). https://quizlet.com/

\section{METODOLOGÍA}

Este estudio descriptivo-cualitativo, parte de la idea de que los estudiantes encuentran más motivante el uso de REA que un ejercicio del libro de texto y/o en PDF. La intención es evaluar 
algunas de las características de estos REA dentro de la población estudiantil y orientar a futuros docentes en la búsqueda de nuevos recursos que fueran interactivos, apropiados y motivantes, siendo empleados de manera sincrónica y asincrónica al mismo tiempo estimular la creatividad, la curiosidad, el diálogo, los intereses, las necesidades.

Para poder identificar los REA que tuvieran estas características, se construyó una encuesta para saber cuáles recursos fueron los más significativos en su proceso de aprendizaje. Esta encuesta intenta describir e indagar opiniones, pensamientos y sentimientos de los estudiantes y qué recursos fueron seleccionados por su gusto, utilidad y preferencia.

Los 50 participantes de esta encuesta $82 \%$ mujeres y $18 \%$ hombres de los cuales 26 son estudiantes que cursan los niveles de Inglés intermedio I y III de diversas licenciaturas de las tres divisiones; Ciencias Básicas e Ingeniería (CBI), Ciencias Biológicas y de la Salud (CBS) y Ciencias Sociales y Humanidades (CSH) de la Universidad Autónoma Metropolitana-Iztapalapa en la Ciudad de México y 24 estudiantes de diversas carreras cursando el nivel básico III del Centro de Idiomas de la Universidad Veracruzana en la Región Veracruz.

Debido a la pandemia, los estudiantes se vieron en la necesidad de regresar a sus lugares de origen, y los profesores tuvieron que adaptarse a dar clases de manera virtual o remota. Los estudiantes se encontraron con problemas de luz eléctrica, acceso a internet, la falta de empleo para poder pagar el internet de su casa, desplazarse a otra localidad para tener acceso a una computadora, entre otros problemas, sin embargo participaron activamente en las tareas que les fueron asignadas porque eran novedosas e interactivas.

A partir de la pandemia y a lo largo de las clases en videoconferencia los estudiantes han externado su preferencia por actividades dinámicas e interactivas en la clase de inglés. Los aprendientes comentaron que este tipo de recursos los motivaban a estudiar con mayor interés los temas expuestos y buscar más recursos similares.

Ante la vasta cantidad de REA que en la actualidad se ofertan se hizo un consenso de qué herramientas pueden coadyuvar a realizar las clases de forma más dinámica e interactiva para promover el aprendizaje de la lengua inglesa. Los recursos utilizados cumplieron cabalmente con los objetivos planteados, brindando apoyo a las habilidades orales, de escritura, de audición y sus competencias tecnológicas.

Cada una de las herramientas ya mencionadas fueron activadas durante esta experiencia. A continuación se presentan los resultados empíricos reportados por los docentes que participaron en esta experiencia.
Los recursos lúdicos o de gamificación Kahoot y Quizizz fueron utilizados para explorar conocimientos previos antes de la presentación del tema ya sea gramatical o cultural y/o para reafirmar los conocimientos adquiridos al finalizar dichas unidades de aprendizaje. La competencia, el incremento del interés, la satisfacción por conseguir los objetivos planteados, la reflexión y el concientizar que el tema no es comprendido en su totalidad, los ayudó a concientizar sobre sus áreas de oportunidad, sus fortalezas y debilidades.

Las habilidades de producción oral y comprensión auditiva fueron apoyadas por los recursos Flipgrid y Edpuzzle. Flipgrid promovió la expresión oral de manera creativa y semi estructurada, ya que el estudiante tenía que elaborar un video corto haciendo preguntas indirectas sobre su ciudad lo cual le permitió que el alumno pensará su respuesta y practicara su pronunciación, entonación y fluidez antes de presentar su video.

Edpuzzle permitió a los estudiantes practicar su comprensión auditiva por medio de videos interactivos, donde el estudiante tienen que responder una serie de preguntas de lo escuchado y visto en el video. Es un recurso invaluable ya que el estudiante puede reproducir el video cuantas veces sea necesario para comprender en el audio, sabiendo su resultado ya que ofrece retroalimentación instantánea en las preguntas de opción múltiple.

Quizlet por su parte, apoyó a la adquisición de vocabulario o de temas gramaticales de forma interactiva y lúdica con sus seis modalidades de enseñanza apoyando a todos los estilos de aprendizaje ya que el alumno escribe, lee, oye y juega en una sola aplicación. La retroalimentación instantánea, el formato y visualización de las actividades promovieron que el aprendiente se motive a seguir compitiendo consigo mismo y con el grupo.

Finalmente, Padlet fomentó la habilidad escrita por medio de un muro donde compartían sus experiencias personales, por medio de los recuerdos de su infancia repasando puntos gramaticales en pasado. Hacían uso de su creatividad a la hora de diseñar y presentarlo en el muro. Otra actividad a realizar en Padlet era leer otros escritos y comentar y/ o hacer preguntas. Esto se volvió un reto porque los jóvenes contaron con problemas desde conectividad, falta de luz y de insumos físicos.

\section{RESULTADOS}

Se escogió como método la encuesta para la recolección de datos por ser un instrumento rápido y de fácil acceso a todos; además su interpretación puede ser mediante gráficos o extractos de opiniones. La encuesta contenía 10 preguntas, 5 preguntas abiertas y 4 de opción múltiple y una de varias opciones. Esta entrevista se elaboró por Google Forms y fue enviada por teléfono (Whatsapp), y la plataforma educativa Google Classroom. La muestra fueron 50 estudiantes 
de 2 universidades en México (Universidad Autónoma Metropolitana-Iztapalapa en la Ciudad de México y Universidad Veracruzana en Veracruz).

En los siguientes gráficos se muestran algunas de las respuestas relacionadas con las variables de esta investigación: gustos, preferencias y utilidad en el aprendizaje. La Pregunta 1 refiere a las herramientas utilizadas durante la contingencia Covid (trabajo a distancia) la gráfica muestra los recursos promovidos por los docentes para hacer más atractivo la enseñanza-aprendizaje. La mayoría de los estudiantes prefirió Quizizz. (preferencia)

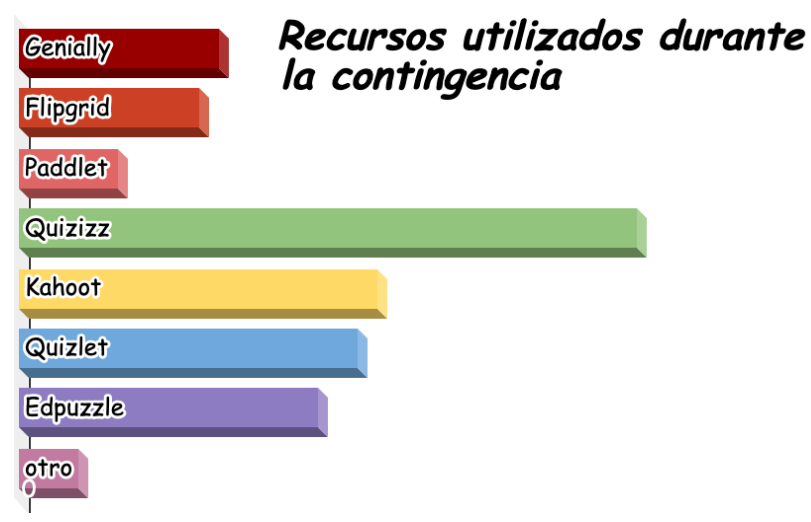

Gráfica 1. Recursos utilizados durante la contingencia.

En la pregunta 2 se refiere a los recursos que los estudiantes han considerado útiles que han aprendido más. El resultado es que las actividades de gamificación son consideradas más útiles y motivantes para desarrollar el trabajo independiente. (utilidad y preferencia)

\section{Recursos considerados útiles por los estudiantes y que facilitaron su aprendizaje}

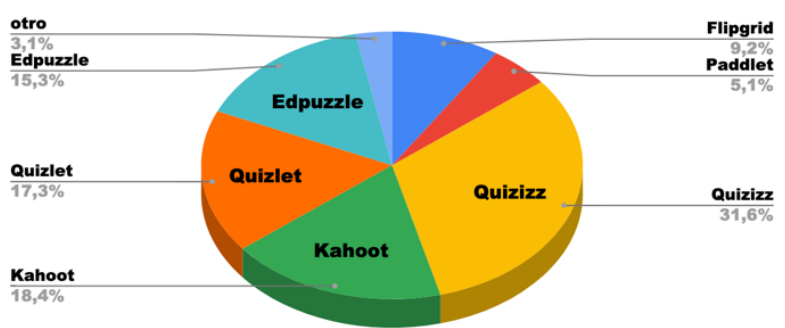

Gráfica 2. Recursos considerados útiles por los estudiantes y que facilitaron su aprendizaje.

La pregunta siete se relaciona con las actividades o recursos que les han servido de manera individual para desarrollar su autonomía. El $80 \%$ de los estudiantes contestó que sí efectivamente les ha servido, y el $20 \%$ dijo que tal vez. Sin embargo, nadie contestó que no les ha servido de apoyo. (utilidad)

\section{Opinión sobre recursos que apoyan al estudio autónomo.}

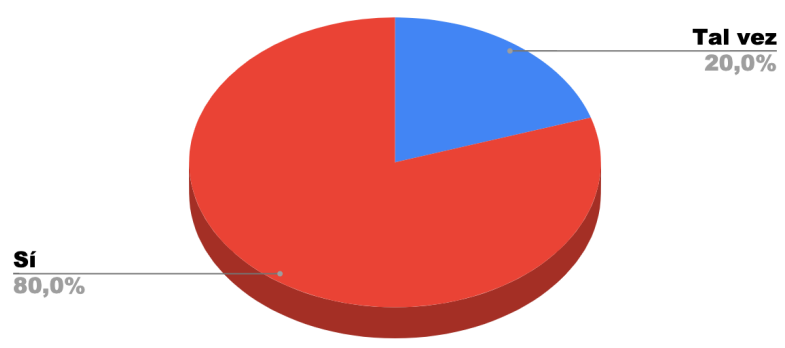

Gráfica 3. Opinión sobre recursos que apoyan al estudio autónomo.

En la pregunta ocho los estudiantes externaron su agrado por un REA más que otros entre sus comentarios externaron que era didáctico, creativo, entretenido, que son recursos visualmente atractivos e interactivos, ayudan a tener mayor atención al inglés y determinan el aprendizaje que llevan, y eso los motivan a estudiar para ganar.

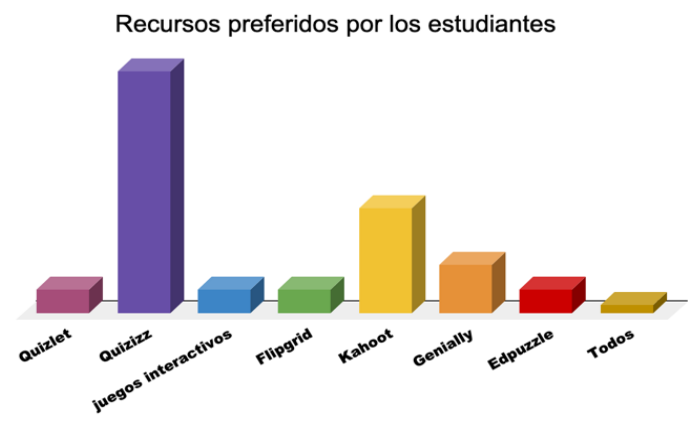

Gráfica 4. Recursos preferidos por los estudiantes. .

La encuesta también arrojó otros hallazgos importantes en este estudio descriptivo. Se indagó si los estudiantes antes de la contingencia habían manejado estas herramientas con sus otras materias y/o profesores. Solamente el 15\% de la muestra, había utilizado Quizizz, 7\% Quizlet y solo el 5\% Kahoot. El $73 \%$ restante desconocía todas estas herramientas.

En otra de las preguntas (pregunta 4), los estudiantes seleccionaron los recursos empleados que les ayudaron a aprender de una forma dinámica, divertida interactiva sin hacer de su aprendizaje algo monótono. Además, sus clases fueron más prácticas y accesibles, puntualizando que los temas más difíciles fueron de mejor entendimiento con la ayuda de estos recursos. Otros externaron que entienden más cuando se explica de una manera más innovadora, resumida y visual a partir de esto, las profesoras diseñaron materiales á la carte en Genially o Quizizz de acuerdo a las necesidades y preferencias de los estudiantes. 
El $100 \%$ de los estudiantes mencionó que sí es importante el conocimiento del profesor para utilizar estas herramientas (pregunta 6). Este hecho lo hace más significativo, ya que trabajar a distancia y desde diferentes lugares hace más complicado el aprendizaje y los únicos medios son una pantalla y las herramientas que el profesor provea.

Se les pidió que agregarán comentarios adicionales pertinentes al uso de estos recursos y los comentarios variaron desde la diversión, la apreciación por el tiempo que le dedican sus maestros a elaborar el material, hasta la sugerencia a otros profesores en aplicar estos REA en otras materias para un mejor aprendizaje. Externan que han aprendido con los recursos en línea de manera independiente y a la hora de tener su video conferencia se aclaran dudas también tienen retroalimentación automática, les exige poner más atención a las actividades a realizar por el temor a equivocarse y perder e incluso otros llegaron a comentar:

"Muy buena dinámica, me gusta mucho porque practico mucho más que cuando son clases presenciales". "Las profesoras nos han apoyado en todo, dudas, comentarios y pronunciación. No nos ha dejado a la deriva, a pesar del tema de la pandemia han estado al pie del cañón y si cometemos errores los sabemos enmendar, pues del error también se aprende ya que estamos siendo más autorreflexivos en nuestro aprendizaje."

\section{CONCLUSIÓN}

Cuando se diseñen materiales de clase, debemos de considerar la potencialidad y las características de los REA en la enseñanza: revisar, combinar, reusar, redistribuir, retener por sus siglas en inglés (Revise, Remix, Reuse, Redistribute and Retain). También es primordial cubrir las necesidades y deseos de los estudiantes ya que; como lo demostró la encuesta, el aprendizaje no fue demeritado o minimizado a pesar de estar en diversas localidades.

Cabe puntualizar que de acuerdo a los resultados se descubrió que si se le dio respuestas a los objetivos. Se promovió la autonomía y la motivación que son elementos fundamentales en el proceso de enseñanza-aprendizaje. No obstante, la investigación también arroja que hace falta instarlos a ser más autónomos y proactivos en su proceso de aprendizaje ya que algunos de ellos externaron que a través de las videoconferencias buscaban videos $u$ otros medios que les diera la explicación gramatical y así poder tener un buen desempeño en las actividades.

Desde otro punto de vista, los estudiantes se mostraron más proactivos, lo que implica un reto a seguir creando materiales que sean amigables en su creación, atractivos e interactivos, lo cual le permite al docente la optimización de tiempo de creación de más material didáctico y su reúso.
Finalmente se puede concluir que la tecnología y el internet son herramientas poderosas que potencializan la enseñanza $y$ ha cambiado el paradigma educativo; por lo tanto es necesario crear materiales digitales para motivar a los estudiantes y apoyarlos a lo largo de su proceso de aprendizaje.

\section{REFERENCES}

\section{REFERENCES}

[1] Baragar, A. (2020). How to use Flipgrid in the classroom. Retrieved from https://www.teachhub.com/how-use-flipgrid-classroom.

[2] Byram, M. y Fleming, M. (2001): Perspectivas interculturales en el aprendizaje de idiomas. Enfoques a través del teatro y la etnografía. Madrid. Cambridge University Press.

[3] Cassany, D (2008). Nativos e inmigrantes digitales en la escuela. Educativa, Ed. Barcelona: Estudios e Investigaciones.

[4] Castillo Niama, M. P., Célleri Quinde, S. P., Rojas Yumisaca, W. G., \& Lara Velarde, A. C. (2019). La motivación intrínseca en el proceso enseñanza - aprendizaje de inglés como lengua extranjera. Ciencia Digital, 3(4.2), 144-159. https://doi.org/10.33262/cienciadigital.v3i4.2.1017

[5] Davies, G., \& Hewer, S. (2012). Introduction to new technologies and how they can contribute to language learning and teaching. Module 1.1 in Davies G. (ed.), Information and Communications Technology for Language Teachers (ICT4LT), Slough, Recuperado de http://www.ict4lt.org/en/en_mod1-1.htm

[6] Deterding, S., Khaled, R., Nacke, L., \& Dixon, D. (2011). Gamification: A definition. Retrieved from http://gamification-research.org/wpcontent/uploads/2011/04/CHI_2011_Gamification_Workshop.pdf

[7] Dommus, V (2010) The use of social networks in educational computergame based foreign language learning. Published by Elsevier Ltd.doi:10.1016/j.sbspro.2010.12.355 Procedia Social and Behavioral Sciences 9 (2010) 1497-1503, Recuperado de https://www.sciencedirect.com/science/article/pii/S1877042810024602

[8] Gardner , R. \& Lambert, W (1985) Attitudes and motivation in Second Language Learning. Rowley, Ma Newbury House.

[9] Gardner, R \& Lambert, W (1959) Motivational Variables in Second Language Acquisition. Canadian Journal of Psychology. 191.-97

[10] González-González, C., \& Mora-Carreño, A. (2015). Técnicas de gamificación aplicadas en la docencia de Ingeniería Informática. Revisión, 8 (1). Consultado el junio 25, 2020, de http://www.aenui.net/ojs/index.php?journal=revision\&page $=$ article\&op=v iew\&path $\% 5 \mathrm{~B} \% 5 \mathrm{D}=152 \&$ path $\% 5 \mathrm{~B} \% 5 \mathrm{D}=301$

[11] García-Salinas, J., Ferreira-Cabrera, A., \& Morales Ríos, S. (2012). Autonomía en el aprendizaje de lenguas extranjeras en contextos de enseñanza mediatizados por la tecnología. Onomázein: Revista de Lingüística, Filología y Traducción de La Pontificia Universidad Católica de Chile, 22(1), 15-50. https://dialnet.unirioja.es/servlet/articulo? codigo $=3982812$

[12] Kapp, K. (2012). The Gamification of Learning and Instruction: GameBased Methods and Strategies for Training and Education. San Francisco: John Wiley \& Sons.

[13] Kazarián, Y., \& Prida-Reinaldo, M. (2014). Activities to motivate students' learning at the English language classes. Revista Habanera de Ciencias Médicas, 13(4), 612-622. http://scielo.sld.cu/scielo.php?script=sci_arttext\&pid=S1729519 X2014000400013\&lng $=$ es\&tlng $=$ en.

[14] Larrenua-Vergara, R. (2014). La motivación en el proceso enseñanza aprendizaje de lenguas extranjeras. Universitat de les Illes Balears, 33. 
https://dspace.uib.es/xmlui/bitstream/handle/11201/145393/Larrenua_Veg ara_Roberto.pdf?sequence $=1 \&$ isAllowed $=\mathrm{y}$

[15] Miao, F. Mishra, S \& McGreal, R. (2016) Open Educational Resources: Policy, Cost and Transformation. UNESCO and Commonwealth of Learning. https://unesdoc.unesco.org/ark:/48223/pf0000244365

[16] Murray, S. (2018). Flipgrid: A modern tech tool to practice the age old art of speaking. Teaching \& Learning, Technology for Learning. Vol. 2 No. 3. Retrieved from https://journal.canadianschoollibraries.ca/flipgrid-amodern-tech-tool-to-practicethe-age-old-art-of-speaking/

[17] Nomass, B.B. (2013). The Impact of Using Technology in Teaching English as a Second Language. English Language and Literature Studies, 3, 111.http://www.ccsenet.org/journal/index.php/ells/article/view/25002

[18] Prensky, M (2008) El papel de la tecnología en la enseñanza y en el aula. Educational Technology. Recuperado de https://aprenderapensar.net/wp-content/uploads/2011/09/El-papel-de-latecnolog\%C3\%ADa-Marc-Prensky.pdf

[19] Prensky, M. (2018) Enseñar a nativos digitales. Biblioteca Innovación Educativa. 2da edición. SM Ediciones. México.

[20] Pizarro-Chacón, G., \& Cordero-Badilla, D. (2013). Las TIC: Una herramienta tecnológica para el desarrollo de las competencias lingüísticas en estudiantes universitarios de una segunda lengua. Revista Electrónica $\begin{array}{llll}\text { Educare, } & 17 & \text { (3), } & \text { 277-292. }\end{array}$ http://www.redalyc.org/comocitar.oa?id=194128798014

[21] Vite-Rodríguez, H. (2012). Ambientes de Aprendizaje. Universidad Autónoma del Estado de Hidalgo. Disponible en: DOI: https://doi.org/10.29057/esh.v2i4.1069

[22] Wiley, D. (2014) "Definición del 'Open' en contenido abierto", sd, < http://opencontent.org/definition/>; John Hilton III, David Wiley, Jared Stein, y Aaron Johnson, "The Four R de Apertura y Análisis ALMS: Marcos para los Recursos Educativos Abiertos," Aprendizaje Abierto: El Diario de Aprendizaje Abierto ya Distancia, vol. 25, no. 1 (septiembre 2014), pp. 3744. $<\mathrm{http}: / / \mathrm{www}$.informaworld.com/smpp/content $\sim$ content $=\mathrm{a} 918784703>$ Anexo

Encuesta

Recursos educativos abiertos para el aprendizaje a distancia que motiven la autonomía.

Edad

Carrera

Lugar de Residencia

Género

1. ¿Qué herramientas has ocupado durante la contingencia lista?

Genially

Flipgrid

Padlet

Quizizz

Kahoot

Quizlet

Edpuzzle

Otro 